Received: 2015.04.19

Accepted: 2015.05 .26

Published: 2015.07.04

\title{
Comparison of $\mathrm{T} 1 \rho$ and $\mathrm{T}^{*}$ Relaxation Mapping in Patients with Different Grades of Disc Degeneration at 3T MR
}

Authors' Contribution: Study Design A Data Collection B Statistical Analysis C Data Interpretation D Manuscript Preparation E Literature Search F Funds Collection G
ABCDEF 1 Xinjuan Zhang

BCDEF 2 Li Yang

BCEF 1 Fei Gao

BC 1 Zhenguo Yuan

BCEF 1 Xiangtao Lin

BCEF 1 Bin Yao

BCE 3 Weibo Chen

BCE 4 Queenie Chan

ABCDEF 1 Guangbin Wang
1 Department of Radiology, Shandong Medical Imaging Research Institute, Shandong University, Jinan, Shandong, P.R. China

2 Department of Radiology, Shanghai Institute of Medical Imaging, Zhongshan Hospital, Fudan University, Shanghai, China

3 Philips Healthcare, Shanghai, China

4 Philips Healthcare, Hong Kong, China
Corresponding Author: Source of support:

Background: $\quad \mathrm{T} 1 \rho$ and $\mathrm{T} 2^{*}$ relaxation times are capable of providing information about early biochemical changes in intervertebral disk degeneration (IVDD). The purpose of this study was to assess and compare T1 $\rho$ and T2* mapping in IVDD with reference to Pfirrmann grade.

Material/Methods: Lumbar sagittal T2-weighted, T1 $\rho$ and T2* relaxation MRI were performed at 3.0T in 42 subjects covering discs L1-L2 to L5-S1. All the discs were morphologically assessed according to the Pfirrmann grade. Regions of interest (ROIs) were drawn over the T1 $\rho$ and T2*mappings, including nucleus pulposus (NP) and annulus fibrosus (AF). Wilcoxon signed rank test, Kruskal-Wallis test, and Spearman rank correlation were performed.

Results: The difference in T1 $\rho$ and $T 2^{*}$ values between NP and AF were highly significant $(P<0.001)$. The trends of decreasing T1 $\rho$ and $T 2^{*}$ values of both NP and AF with increasing Pfirrmann grades was significant $(P<0.01)$, particularly between Pfirrmann grade II and III $(P<0.001)$, whereas T2* mapping was additionally able to detect changes in the AF between Pfirrmann grade I and II $(P<0.05)$. Pfirrmann grades were inversely significantly correlated with both T1 $\rho$ and T2* values in the NP $(r=-0.69, P<0.001 ; r=-0.56, P<0.001)$ and AF $(r=-0.45, P<0.001$; $r=-0.26, P<0.001)$.

Conclusions: The process of IVDD can be detected by T1 $\rho$ and $T 2^{*}$ mapping, particularly at early stage, and both methodologies displayed roughly comparable performance.

MeSH Keywords: $\quad$ Early Diagnosis • Intervertebral Disc Degeneration • Lumbar Vertebrae • Magnetic Resonance Imaging

Full-text PDF: http://www.medscimonit.com/abstract/index/idArt/894406

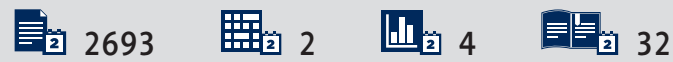




\section{Background}

The prevalence of low back pain (medscimonit-21-1934) ranges from $6.0 \%$ to $15.7 \%$ across the world; it accounts for $49.6 \%$ of the global disability burden and causes an enormous economic impact [1-3]. It was estimated that $41.8 \%$ of LBP can be attributed to intervertebral disk degeneration (IVDD) [4]. Several radiographic findings, such as internal disk dehydration, end-plate sclerosis, and loss of disk height, have been identified on conventional magnetic resonance imaging (MRI) in patients with IVDD. Pfirrmann et al. described a grading system to assess the morphologic changes of IVDD based on standard T2-weighted MRI for research and clinical purposes [5]. Although Pfirrmann grading system is useful, it is subjective and insufficient to detect early-onset IVDD [6]; thus, quantification of the degeneration process is needed to promote disc health evaluation [7].

Over the past decade, new quantitative MRI techniques such as T2- [8-10], T1p- [6,11,12], T2*-relaxation time [10,13,14], diffusion-weighted imaging [15], spectroscopy [16], and delayed gadolinium enhanced MRI [17] have been explored in hopes of revealing early biochemical changes in IVDD, elucidating the degeneration progress, and possibly helping clinicians initiate timely biologic therapies with injected growth factors, genetic material, and stem cells $[18,19]$. In T1 $\rho$ sequence, a "spin lock" pulse is applied to the magnetization transversely and $T 1 \rho$ relaxation time is considered to be sensitive to protons on macromolecules such as glycosaminoglycan (GAG) [20], providing a potential to detect the loss of proteoglycan in IVDD [10]. In vitro studies have reported a correlation between T1 $\rho$ and GAG content in the nucleus pulposus (NP) $[21,22]$. Several investigators have found that $\mathrm{T} 1 \rho$ is correlated with Pfirrmann degenerative grade $[6,8,12,21]$ and clinical symptoms assessed by health questionnaires $[16,23]$. $T 2^{*}$ relaxation times provide information regarding the spatial macromolecule architecture in conjunction with water molecule mobility [7], which has been reported to be a reliable tool for quantitative assessment of various cartilaginous tissues [24-26]. Recent studies have established the relationship between $\mathrm{T}^{*}$ value and Pfirrmann scoring system, as well as macroscopic and histological grading in IVDD, where a decrease in $\mathrm{T}^{*}$ relaxation time was significantly correlated with a higher degree of disc degeneration $[10,13]$. Animal and human cadaveric studies showed a good correlation between $T 2^{*}$ relaxation time and GAG content throughout the discs, as well as between $\mathrm{T}^{*}$ relaxation time and mechanical health $[7,13,14]$. However, little is known about the relative performance of $\mathrm{T} 1 \rho$ and $\mathrm{T} 2^{*}$ relaxation times in their assessment of disc degeneration. The purpose of the current in vivo 3.0-T MRI study was to assess and compare T1 $\rho$ and $\mathrm{T}^{*}$ relaxation time measurements in IVDD with reference to the morphological Pfirrmann grading systems.

\section{Material and Methods}

This prospective study was approved by our institute's research ethics committee, and written informed consent was obtained from each participant prior to the study.

\section{Subjects}

From October 2013 to September 2014, 50 consecutive subjects with single or recurrent episodes of low back pain presented initially to the outpatient spine clinic and then were referred for a lumbar MRI. Inclusion criteria were patients with single or recurrent episodes of low back pain, and radiologic screening and conventional MRI confirmation of no other spine diseases except disc degeneration. Exclusion criteria were a body mass index greater than 25 , spinal malignancy, spine fractures, spinal infection, lumbar scoliosis, sacroiliac arthritis, rheumatoid arthritis, metabolic bone disease, previous spine surgery or interventional treatment, and contraindications for MR imaging.

\section{MR imaging protocol}

MRI was performed in the morning for all subjects to reduce the potential influence of diurnal variation in intervertebral disks. All MRI examinations were performed by a 3.0T scanner (Achieva, Philips Healthcare, Best, the Netherlands) and a dedicated 15-channel SENSE spine coil. First, conventional sagittal T2-weighted images were acquired by using a turbo spin echo (TSE) sequence with the following parameters: $\mathrm{TR} / \mathrm{TE}=2500 \mathrm{~ms} / 90 \mathrm{~ms}$, flip angle $=90^{\circ}$, field of view $=220 \mathrm{~mm}$, $\mathrm{NSA}=2$, and scan time $=1$ min $40 \mathrm{~s}$. Next, a series of sagittal $\mathrm{T} 1 \rho$ and T2* quantification sequences were performed. The T1 $\rho$-weighted images were obtained with a 3D balanced fast field echo ( $b-F F E)$ sequence with the following parameters: $\mathrm{TR} / \mathrm{TE}=4.8 \mathrm{~ms} / 2.4 \mathrm{~ms}$, flip angle $=50^{\circ}, \mathrm{NSA}=1$. Five subsequent T1 $\rho$-weighted scans were performed with spin lock durations of $0,10,20,30$, and $40 \mathrm{~ms}$, spin-lock frequency of $500 \mathrm{~Hz}$, and with scan time of $1 \mathrm{~min} 9 \mathrm{~s}$ for each scan. The $\mathrm{T} 2^{*}$ relaxation time measurements were acquired by a fast field echo (FFE) sequence with the following parameters: TR/TEs $=310 \mathrm{~ms} / 5.1$; 10.0; 14.9; $19.8 ; 24.7 \mathrm{~ms}$, flip angle $=25^{\circ}, \mathrm{NSA}=4$, and acquisition time $=2 \min 46 \mathrm{~s}$. To guarantee the same in-plane and outof-plane resolution for better comparability between the T1 $\rho$ and $\mathrm{T}^{*}$ sequences, the following parameters were kept consistent: field of view $=220 \mathrm{~mm}$, matrix $=432 \times 432$, slice thickness $=5 \mathrm{~mm}$, slices $=9$, voxel size $=0.57 \times 0.57 \times 5.00 \mathrm{~mm}^{3}$.

\section{Image post-processing and analysis}

MR images were transferred to a workstation (Extended MR Workspace, version 2.6.3.2, Philips Healthcare). Two radiologists (with 5 and 10 years of experience in musculoskeletal 
radiology) independently assessed the five lumbar discs in each subject on the mid-sagittal T2-weighted images according to the Pfirrmann grading system [5]: grade I, homogeneous shape, bright hyperintense white signal intensity, normal disc height, and clear distinction of annulus fibrosus (AF) and NP; grade II, nonhomogeneous shape with or without horizontal gray bands, hyperintense white signal, normal disc height, clear distinction of AF and NP; grade III, nonhomogeneous shape, intermediate gray signal intensity, normal or slightly decreased height, and unclear distinction of AF and NP; grade IV, nonhomogeneous shape, hypointense dark gray signal intensity, normal to moderate decrease in height, and lost distinction of AF and NP; and grade V, same as grade IV but collapsed disc space. In cases of discrepancy, a consensus reading was obtained.

The T1 $\rho$-weighted images were fitted on a pixel by pixel basis to the exponentially decaying T1 $\rho$ function using IDL 6.3 (ITT Visual Information Solutions, Boulder, CO) to generate a $T 1 \rho$ relaxation map, and the $T 2^{*}$-weighed image analyses were conducted by using Image J software (National Institutes of Health, Bethesda, MD) [19]. Regions of interest (ROIs) were set manually on the T1 $\rho$ and $T 2^{*}$ mappings with reference to the $\mathrm{T} 2$ images. The ROIs were drawn on the NP, anterior AF (AAF) and posterior AF (PAF). Values of AAF and PAF were averaged as the value for AF. Care was taken to exclude an apparent tear of annulus in the ROls. The ROI sizes for NP were $10 \sim 55 \mathrm{~mm}^{2}$, while the ROI sizes for AF (anterior and posterior) were 10 45 $\mathrm{mm}^{2}$. All the measurements were performed twice by a single observer with an interval of four weeks.

\section{Statistical analysis}

The intraclass correlation coefficients (ICC) with $95 \%$ confidence intervals $(95 \% \mathrm{Cls})$ was used to assess the reproducibility in two measurements of $\mathrm{T} 1 \rho$ and $\mathrm{T} 2$ * relaxation times, and ICC values that were $\geq 0.75$ were considered excellent agreement [27]. The differences of T1 $\rho, T 2 *$ relaxation times between NP and $\mathrm{AF}$ were tested using the Wilcoxon signed rank test, as well as between AAF and PAF. Kruskal-Wallis test and posthoc tests were applied to compare the difference in T1 $\rho$ and T2 values of various disc degeneration grades of NP and AF. Associations between T1 $\rho, T 2 *$ relaxation times and Pfirrmann grade were tested using Spearman rank correlation. Correlations were considered strong for $r>0.7$, moderate for $0.5<r \leq 0.7$, and weak for $r \leq 0.5$ [21]. All statistical analysis was performed by PASW Statistics software (v17.0, SPSS Inc., Chicago, IL, USA), $P<0.05$ was considered statistically significant.

\section{Results}

\section{General data}

Of the 50 patients recruited during the study period, 8 patients were excluded for the following reasons: body mass index greater than $25(n=3)$; sacroiliac arthritis $(n=2)$, and motion artifacts $(n=3)$. A total of 42 subjects ( 30 men, 12 women; rang, 21 80 years; mean age, 44.2 years) with 210 intervertebral lumbar disks from $L 1-2$ to $L 5-S 1$ were included in this study.

The distribution of the T1 $\rho$ and $\mathrm{T}^{*}$ values of the 210 intervertebral disks with respect to the Pfirrmann grading is provided in Table 1 and Figure 1A, 1B. Representative T1 $\rho$ and T2* maps of subjects with different grades of degeneration are shown in Figure 2. In the reliability of measurements, ICCs with $95 \%$ Cls for each technique at different ROls are shown in Table 2; the reproducibility between the two measurements was excellent $(\mathrm{ICC}=0.909 \sim 0.969)$. $\mathrm{T} 1 \rho$ relaxation time ranged from 73.8 to $235.1 \mathrm{~ms}$ for NP, and from 55.7 to $173.8 \mathrm{~ms}$ for $\mathrm{AF}$, while T2* relaxation times ranged from 9.6 to $185.8 \mathrm{~ms}$ for NP, and from 12.0 to $52.7 \mathrm{~ms}$ for AF. The difference in T1 $\rho$ and $T 2^{*}$ values between NP and AF were highly significant (both $P<0.001$ ), and the difference in $\mathrm{T} 1 \rho$ and $\mathrm{T} 2^{*}$ values between $\mathrm{AAF}$ and PAF were also highly significant (both $P<0.01$ ).

\section{T1 $\rho$ and T2* values versus Pfirrmann grade}

Boxplots of $\mathrm{T} 1 \rho$ and $\mathrm{T} 2^{*}$ values in NP and $\mathrm{AF}$ are shown in Figure 3, respectively, which showed trends of decreasing T1 $\rho$ and $\mathrm{T} 2^{*}$ values with increasing Pfirrmann grade. The KruskalWallis test showed a significant difference in T1 $\rho$ and $T 2^{*}$ relaxation times of NP and AF among the Pfirrmann grade groups $(P<0.01)$. For both $\mathrm{T} 1 \rho$ (Figure $3 \mathrm{~A})$ and $\mathrm{T} 2^{*}$ relaxation times (Figure $3 \mathrm{~B}$ ) in the NPs, a significant difference was found between Pfirrmann grade II and Pfirrmann grades greater than II $(P<0.001)$, especially between Pfirrmann grade II and III, and the difference was not statistically significant between Pfirrmann grade I and II $(P>0.05)$, nor between Pfirrmann grade IV and $V(P>0.05)$. For AFs, the T1 $\rho$ (Figure $3 C$ ) values also showed Pfirmann grade II was significantly different from Pfirrmann grades higher than II $(P<0.001)$, particularly notable between Pfirmann grades II and III; the T2* values (Figure 3D) demonstrated significant difference between Pfirrmann grade I and all the other grades $(P<0.05)$.

\section{Correlations}

Spearman correlation analysis demonstrated that Pfirrmann grades were inversely significantly correlated with both $T 1 \rho$ and $2^{*}$ values in the NP $(r=-0.69, P<0.001$ (Figure $4 \mathrm{~A}) ; r=-0.56$, $P<0.001$ (Figure 4B)) and $\mathrm{AF}(r=-0.45, P<0.001$ (Figure $4 \mathrm{C}$ ); $r=-0.26, P<0.001$ (Figure 4D)). 
Table 1. T1 $\rho$ and $\mathrm{T}^{*}$ relaxation times on intervertebral disks with Pfirrmann grades.

\begin{tabular}{|c|c|c|c|c|c|c|c|c|c|c|}
\hline \multirow{2}{*}{$\begin{array}{l}\text { Pfirrmann } \\
\text { grade }\end{array}$} & \multirow{2}{*}{ n (\%) } & & \multicolumn{4}{|c|}{$\mathrm{T} 1 \rho$ relaxation time $(\mathrm{ms})$} & \multicolumn{4}{|c|}{$\mathrm{T}^{*}$ relaxation time $(\mathrm{ms})$} \\
\hline & & & NP & AAF & PAF & AF & NP & AAF & PAF & AF \\
\hline \multirow{3}{*}{ I } & \multirow{3}{*}{$41(19.5)$} & Median & 172.3 & 107.6 & 119.7 & 114.3 & 83.8 & 27.6 & 33.3 & 31.6 \\
\hline & & Mean & 177.3 & 113.9 & 120.9 & 117.4 & 85.8 & 28.7 & 35.9 & 32.3 \\
\hline & & SD & 36.0 & 25.9 & 27.0 & 21.3 & 41.7 & 10.4 & 13.7 & 9.4 \\
\hline \multirow{3}{*}{ II } & \multirow{3}{*}{$68(32.4)$} & Median & 170.0 & 114.4 & 120.1 & 119.6 & 59.8 & 25.3 & 27.4 & 27.3 \\
\hline & & Mean & 172.2 & 115.2 & 125.8 & 120.5 & 63.3 & 26.7 & 29.4 & 28.1 \\
\hline & & SD & 31.9 & 22.2 & 23.8 & 18.2 & 27.4 & 8.5 & 10.9 & 8.1 \\
\hline \multirow{3}{*}{ III } & \multirow{3}{*}{$53(25.3)$} & Median & 121.9 & 99.3 & 102.5 & 100.2 & 40.8 & 26.6 & 26.1 & 26.4 \\
\hline & & Mean & 128.8 & 102.3 & 105.1 & 103.7 & 42.7 & 27.3 & 26.2 & 27.2 \\
\hline & & SD & 30.1 & 22.1 & 23.1 & 20.0 & 14.5 & 7.3 & 7.5 & 7.3 \\
\hline \multirow{3}{*}{ IV } & \multirow{3}{*}{$41(19.5)$} & Median & 99.6 & 91.6 & 98.9 & 92.9 & 33.5 & 25.2 & 25.1 & 24.8 \\
\hline & & Mean & 105.7 & 94.6 & 101.2 & 97.9 & 33.9 & 24.6 & 25.8 & 25.3 \\
\hline & & SD & 21.5 & 16.6 & 17.8 & 15.2 & 12.1 & 7.1 & 7.3 & 6.2 \\
\hline \multirow{3}{*}{ V } & \multirow{3}{*}{$7(3.3)$} & Median & 100.8 & 73.1 & 79.3 & 82.2 & 24.3 & 21.6 & 25.9 & 24.4 \\
\hline & & Mean & 99.1 & 77.6 & 85.4 & 81.5 & 26.1 & 20.4 & 24.2 & 22.3 \\
\hline & & SD & 12.2 & 17.1 & 18.7 & 14.4 & 11.2 & 5.1 & 5.4 & 4.4 \\
\hline
\end{tabular}

NP - nucleus pulposus; AAF - anterior annulus fibrosus; PAF - posterior annulus fibrosus; AF - annulus fibrosus; \# The percentages of patients assigned to each of the Pfirrmann grades on the basis of the T2-weighted image.

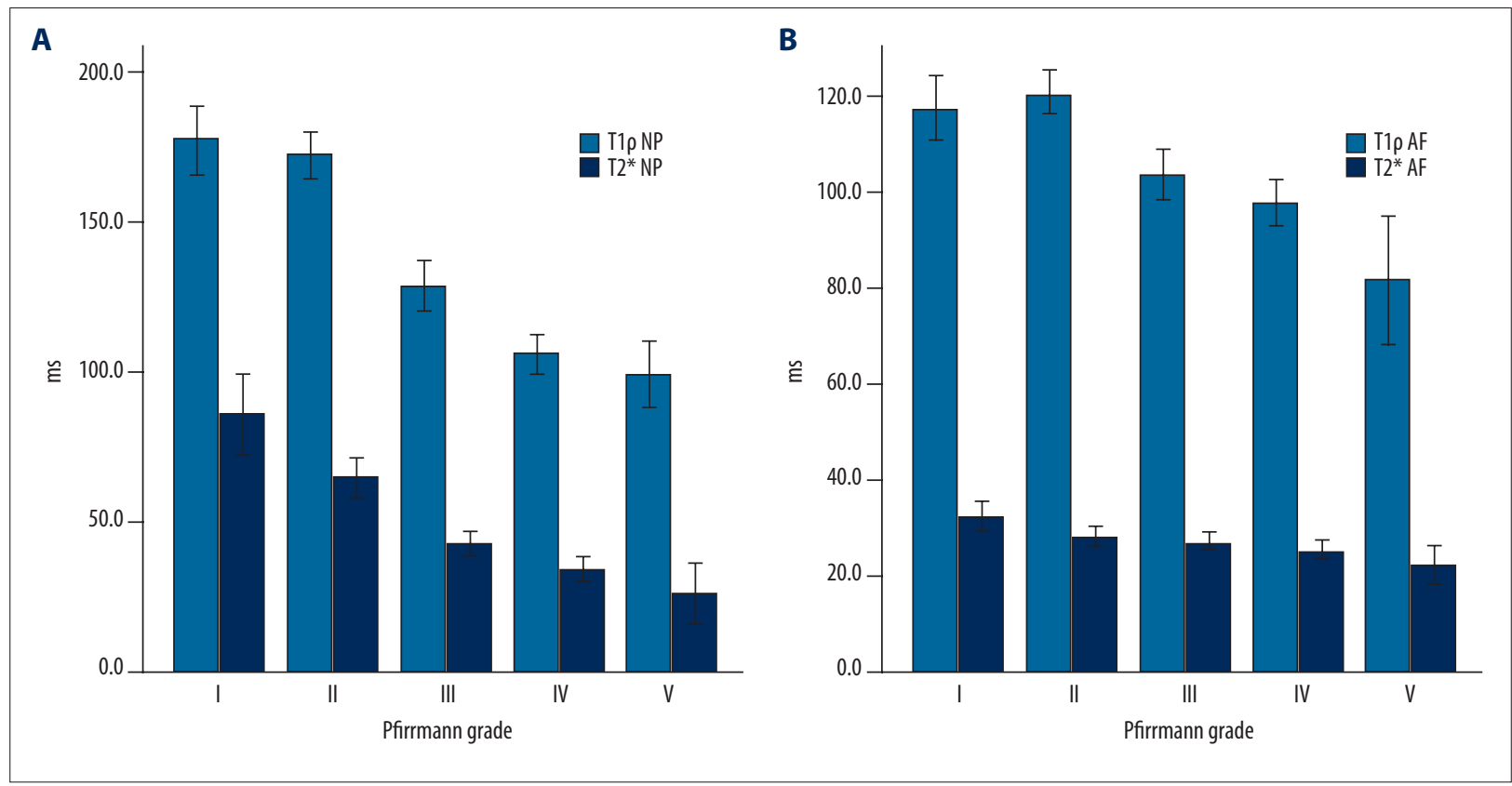

Figure 1. The distribution of the T1 $\rho$ and $T 2^{*}$ values of nucleus pulposus (NP) (A) and annulus fibrosus (AF) (B) with respect to the Pfirrmann grades. 


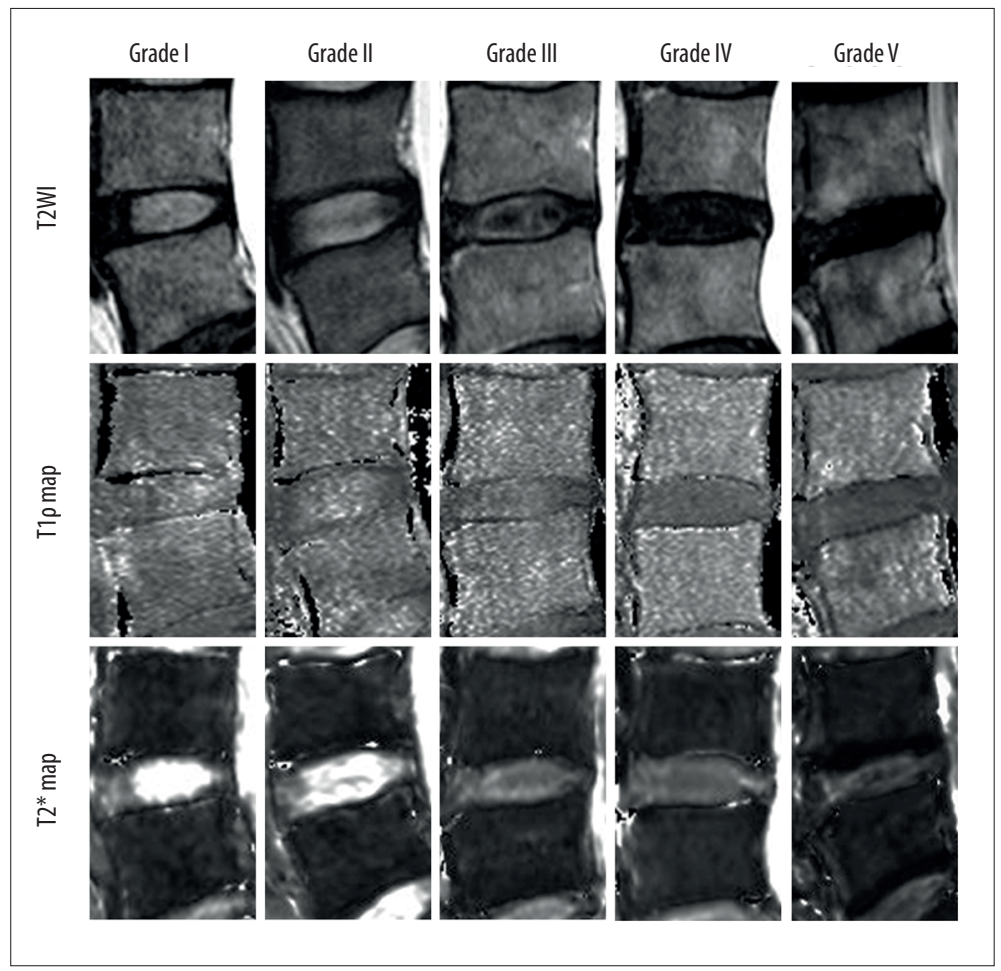

Figure 2. T2-weighted images and corresponding $\mathrm{T} 1 \rho$ and $\mathrm{T} 2^{*}$ maps in human intervertebral disk degeneration related to Pfirrmann grades.

Table 2. The intraclass correlation coefficients (ICC) with $95 \%$ confidence intervals (Cls) for intraobserver analysis of T1 $\rho$ and T2* mapping.

\begin{tabular}{ccccc}
\hline & & T1 $\rho$ mapping & \multicolumn{2}{c|}{ T2* mapping } \\
\hline ROIs & ICC & $\mathbf{9 5 \% ~ C l}$ & ICC & $\mathbf{9 5 \% ~ C l}$ \\
\hline AAF & 0.955 & $0.941-0.966$ & 0.969 & $0.959-0.976$ \\
\hline PAF & 0.932 & $0.886-0.950$ & 0.937 & $0.893-0.957$ \\
\hline
\end{tabular}

$\mathrm{ROI}$ - regions of interest; NP - nucleus pulposus; AAF - anterior annulus fibrosus; PAF - posterior annulus fibrosus.

\section{Discussion}

In this study, significant negative correlations were observed between the $T 1 \rho, T 2^{*}$ relaxation times and the Pfirrmann grades. Moreover, both methodologies displayed roughly comparable performance. To the best of our knowledge, this is the first comparison of T1 $\rho$ and $T^{*}$ relaxation times at $3 \mathrm{~T} M R$ in patients experiencing low back pain with different grades of disc degeneration.

The human intervertebral disc is composed of the central NP, the peripheral $\mathrm{AF}$, and the endplates connecting to the vertebrae. Normally, the NP is gelatinous, well-hydrated, and mainly consists of proteoglycans enclosed by the type II collagen network, which produces a high internal hydrostatic pressure that allows the disk to support compressive load in the spine. The $\mathrm{AF}$ is characterized by concentric lamellae primarily of bundles of type I and III collagen, which serves as the limiting capsule of the NP $[28,29]$. In a healthy young adult, proteoglycan accounts for about $50 \%$ of the NP and $10-20 \%$ of the AF dry weight, while collagen accounts for approximately $15-20 \%$ of the NP and $65-70 \%$ of the AF dry weight [30]. At the earliest stages of IVDD, the proteoglycan degrades and its content decreases; thus, it is of great importance to probe this change in subjects with IVDD noninvasively. However, conventional MRIs are incapable of estimating the proteoglycan concentration in the disc [31]. New MR techniques such as T1 $\rho$ and T2* mapping provide information of biochemical composition in disc and thus allow detection of early changes in IVDD.

T1 $\rho$ relaxation time was obtained by using spin-lock radiofrequency field, which is related to slow motional interactions between macromolecules of extracellular matrix and bulk water [32]. Biochemical analysis on cadaveric human discs revealed 

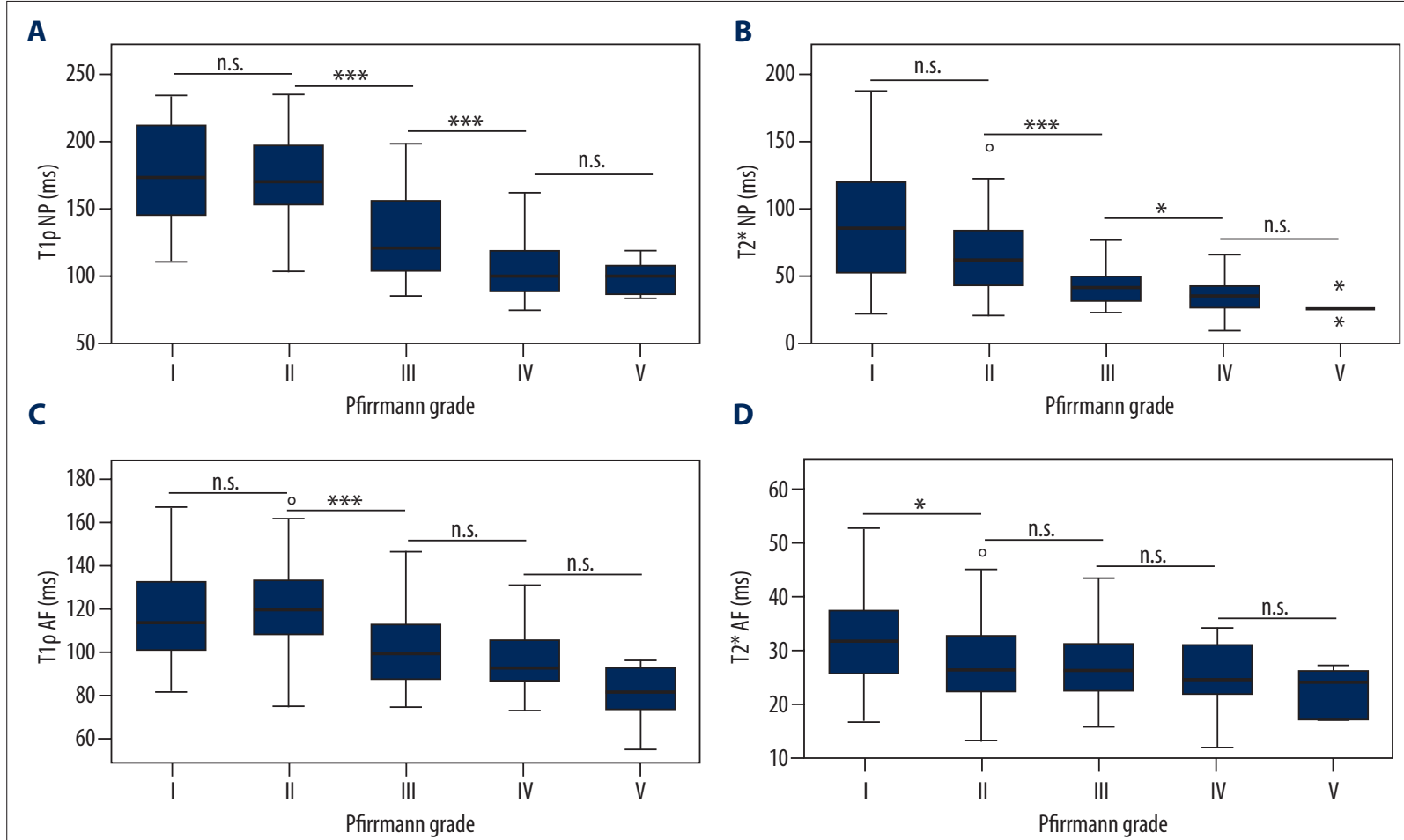

Figure 3. Box plots show trends of decreasing $T 1 \rho$ and $T 2^{*}$ values of nucleus pulposus (NP) and annulus fibrosus (AF) with increasing Pfirrmann grades, and great T1 $\rho$ and T2* value decrease between Pfirrmann grades II and III. (A) T1 $\rho$ relaxation time of NP versus disc degeneration grading; (B) T2* relaxation time of NP versus disc degeneration grading; (C) T1 $\rho$ relaxation time of AF versus disc degeneration grading; (D) T2* relaxation time of AF versus disc degeneration grading. ${ }^{*} P<0.05$; ${ }^{* * *} P<0.001$; n.s. not significant.

that the T1 $\rho$ relaxation time was strongly correlated with proteoglycan content and was moderately correlated with water content $[21,22]$. Our results showed a decreasing trend in T1 $\rho$ values of both NP and AF with the increase of Pfirrmann grade, and the association between T1 $\rho$ values of NP and AF and Pfirmann grade was significant $(r=-0.69, P<0.001 ; r=-0.45$, $P<0.001$ ), which is in agreement with previous reports that T1 $\rho$ values of AF decline significantly over disc degeneration [9] and that T1 $\rho$ values of NP are moderately correlated with Pfirrmann grade I-V disks of patients with low back pain [6].

T2* relaxation embraces the intrinsic "true" $\mathrm{T} 2$ relaxation and additional relaxation because of magnetic inhomogeneities, and is sensitive to the spatial macromolecule architecture and its interaction with water molecule mobility [7]. Recent studies have demonstrated the low-to-moderate correlation between $\mathrm{T}^{*}$ value and Pfirmann grade, as well as $\mathrm{T} 2^{*}$ value and GAG content in IVDD $[10,13]$. In the present study, T2* relaxation times on NP were significantly higher than AF, and both $\mathrm{T} 2{ }^{*}$ values were negatively correlated with Pfirmann grades $(r=-0.56, P<0.001 ; r=-0.26, P<0.001)$. Welsch et al. reported an increase in $T 2^{*}$ values from the AAF to the NP and the subsequent decline from the NP to the PAF, and a decrease in $\mathrm{T}^{*}$ values on NP and PAF was significantly correlated with a higher Pfirrmann grade $(r=-0.220 \sim-0.334, p<0.01)$ in patients experiencing low back pain [10], which was comparable to our results.

With regard to the discernment of the single Pfirrmann grade, $\mathrm{T} 1 \rho$ and $\mathrm{T} 2^{*}$ mapping displayed roughly comparable performance. In particular, both $\mathrm{T} 1 \rho$ and $\mathrm{T}^{*}$ mapping seemed to be able to differentiate between Pfirrmann grade II and III, as well as between Pfirrmann grade III and IV. A relatively larger decline in T1 $\rho$ and $\mathrm{T}^{*}$ values between Pfirmann grades II and III was observed, which probably was due to rapid degeneration of lumbar intervertebral discs confirmed in an animal model by Zhou et al. [6]. Moreover, T2* values of AF demonstrated a significant difference between Pfirrmann grade I and II, indicting $\mathrm{T}^{*}$ relaxation time might not only be sensitive to GAG content, but also to collagen integrity [10].

Probing the early changes in the degeneration process of intervertebral discs would be beneficial for timely developing and applying new therapeutics $[18,19]$. The Pfirrmann classification cannot provide quantitative and objective evaluation of early IVDD. This deficit may be filled by $T 1 \rho$ and $T 2^{*}$ relaxation time measurements. Particularly in the longitudinal follow-up 


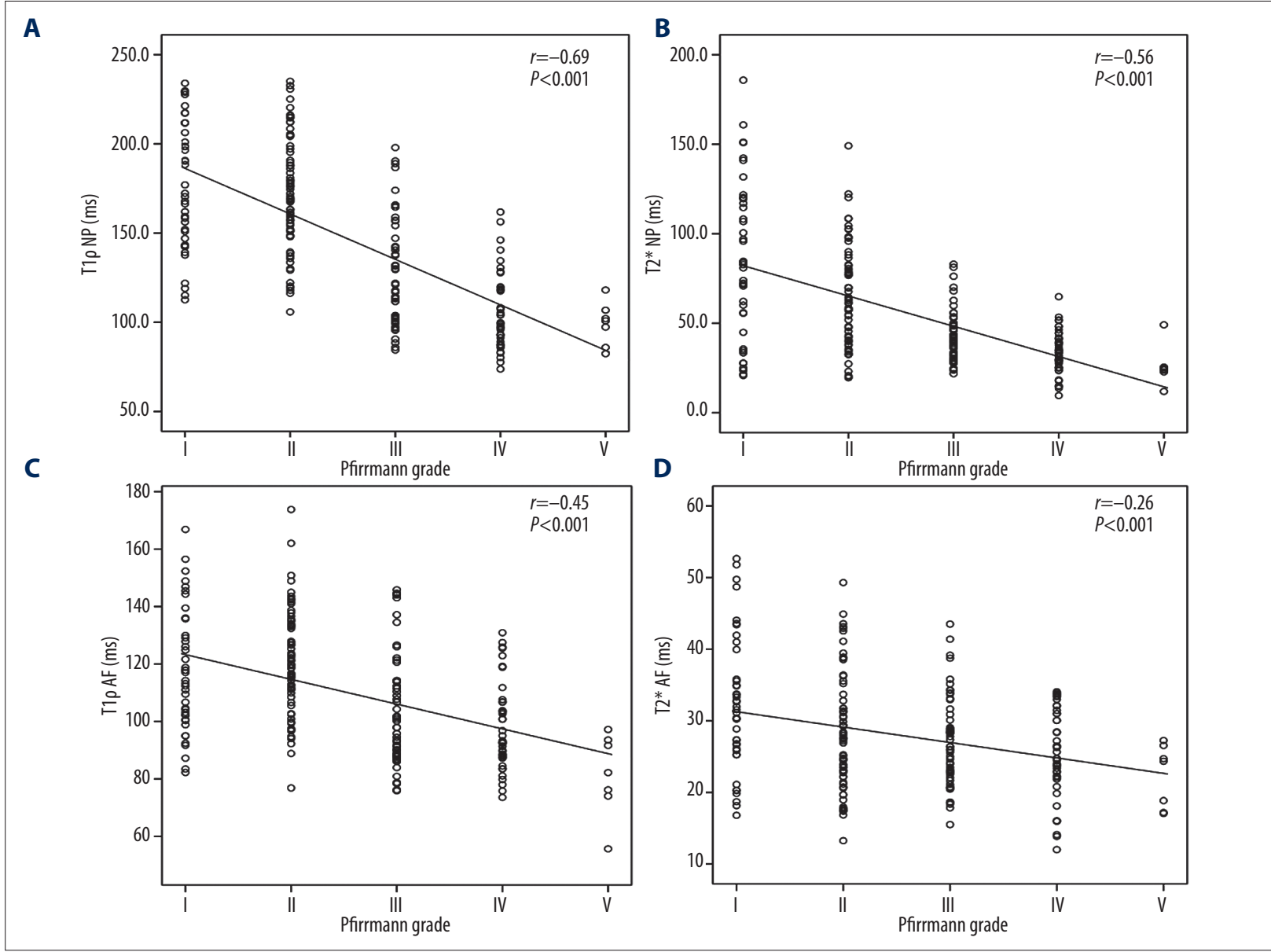

Figure 4. Scatter plots of $\mathrm{T} 1 \rho$ and $T 2^{*}$ values in nucleus pulposus (NP) and annulus fibrosus (AF) according to the Pfirrmann grades. (A) Correlation between the T1 $\rho$ relaxation time of NP and degenerative grades. (B) Correlation between the T2* relaxation time of NP and degenerative grades. (C) Correlation between the T1 $\rho$ relaxation time of AF and degenerative grades.

(D) Correlation between the $\mathrm{T}^{*}$ relaxation time of $\mathrm{AF}$ and degenerative grades.

of patients with IVDD, T1 $\rho$ and $T 2 *$ values might provide a reliable quantification of longitudinal biochemical changes of degeneration [10]. T1 $\rho$ and T2* mapping in this work showed roughly comparable performance in assessing the biochemical content of the IVDD. However, T1 $\rho$-weighted imaging is not yet easily applicable on standard MRI scanners, while T2* mapping could be routinely available on a clinical MR scanner [11]. Additionally, the acquisition time on $\mathrm{T} 2^{*}$ mapping was almost half of that on the T1 $\rho$ mapping ( $2 \min 46 \mathrm{~s} v s .5 \min 45 \mathrm{~s}$ ). Hence, $T 2^{*}$ mapping is more promising than $\mathrm{T} 1 \rho$ mapping in diagnosing IVDD in routine clinical practice.

There are several limitations in this study. On the one hand, the present results were not verified by histological and biochemical findings, partly because of inability in obtaining specimens from human subjects. On the other hand, the relation between the $\mathrm{T} 1 \rho$ or $\mathrm{T} 2^{*}$ mapping and physical symptoms was not investigated, particularly the degree and duration of LBP, as various causes account for discogenic LBP (such as innervations and tear of the annulus) and the mechanism by which IVDD leads to LBP remains unclear [23]. Besides, comparison of the sex difference in $T 1 \rho$ values at different levels showed a significant lower value in the female discs at L3-L4 and L4-L5 discs of the NP, and at L3-L4 disc of the AF [12], but the assessments of $T 1 \rho$ and $T 2^{*}$ relation times according to the anatomic level of each disc and sex were not performed in this study because the size of women was small and the emphasis of current study was on the relative performance of T1 $\rho$ and $\mathrm{T}^{*}$ mapping. To draw more detailed and definitive conclusions during the process of disc degeneration, further studies including a large number of patients with multiple followup MRI and in vitro investigations are needed.

\section{Conclusions}

In conclusion, our results show that the $\mathrm{T} 1 \rho$ and $\mathrm{T} 2^{*}$ values significantly and negatively correlate with the Pfirrmann grades of disc 
degeneration, and both methodologies displayed roughly comparable performance. $\mathrm{T} 1 \rho$ and $\mathrm{T} 2^{*}$ relaxation time measurements may promote the detection of IVDD at a relatively early stage.

\section{References:}

1. Hoy D, March L, Brooks $P$ et al: The global burden of low back pain: estimates from the Global Burden of Disease 2010 study. Ann Rheum Dis, 2014; 73: 968-74

2. March L, Smith EU, Hoy DG et al: Burden of disability due to musculoskeletal (MSK) disorders. Best Pract Res Clin Rheumatol, 2014; 28: 353-66

3. Misterska E, Jankowski R, Glowacki M: Chronic pain coping styles in patients with herniated lumbar discs and coexisting spondylotic changes treated surgically: Considering clinical pain characteristics, degenerative changes, disability, mood disturbances, and beliefs about pain control. Med Sci Monit, 2013; 19: 1211-20

4. DePalma MJ, Ketchum JM, Saullo T: What is the source of chronic low back pain and does age play a role? Pain Med, 2011; 12: 224-33

5. Pfirrmann CW, Metzdorf A, Zanetti M, Hodler J, Boos N: Magnetic resonance classification of lumbar intervertebral disc degeneration. Spine (Phila Pa 1976), 2001; 26: 1873-78

6. Zhou Z, Jiang B, Zhou Z et al: Intervertebral disk degeneration: T1rho MR imaging of human and animal models. Radiology, 2013; 268: 492-500

7. Ellingson AM, Mehta H, Polly DW et al: Disc degeneration assessed by quantitative $T 2^{*}$ (T2 star) correlated with functional lumbar mechanics. Spine (Phila Pa 1976), 2013; 38: E1533-40

8. Marinelli NL, Haughton VM, Anderson PA: T2 relaxation times correlated with stage of lumbar intervertebral disk degeneration and patient age. Am J Neuroradiol, 2010; 31: 1278-82

9. Wang YX, Zhao F, Griffith JF et al: T1rho and T2 relaxation times for lumbar disc degeneration: an in vivo comparative study at 3.0-Tesla MRI. Eur Radiol, 2013; 23: 228-34

10. Welsch GH, Trattnig S, Paternostro-Sluga T et al: Parametric T2 and T2* mapping techniques to visualize intervertebral disc degeneration in patients with low back pain: initial results on the clinical use of 3.0 Tesla MRI. Skeletal Radiol, 2011; 40: 543-51

11. Blumenkrantz G, Li X, Han ET et al: A feasibility study of in vivo T1rho im aging of the intervertebral disc. Magn Reson Imaging, 2006; 24: 1001-7

12. Zobel BB, Vadala G, Del Vescovo R et al: T1rho magnetic resonance imaging quantification of early lumbar intervertebral disc degeneration in healthy young adults. Spine (Phila Pa 1976), 2012; 37: 1224-30

13. Detiger SE, Holewijn RM, Hoogendoorn RJ et al: MRI T2* mapping correlates with biochemistry and histology in intervertebral disc degeneration in a large animal model. Eur Spine J, 2014 [Epub ahead of print]

14. Ellingson AM, Nagel TM, Polly DW et al: Quantitative T2* (T2 star) relaxation times predict site specific proteoglycan content and residual mechanics of the intervertebral disc throughout degeneration. J Orthop Res, 2014; 32: $1083-89$

15. Zhang W, Ma X, Wang Y et al: Assessment of apparent diffusion coefficient in lumbar intervertebral disc degeneration. Eur Spine J, 2014; 23: 1830-36

16. Zuo J, Joseph GB, Li X et al: In vivo intervertebral disc characterization using magnetic resonance spectroscopy and T1rho imaging: association with discography and Oswestry Disability Index and Short Form-36 Health Survey. Spine (Phila Pa 1976), 2012; 37: 214-21

\section{Statement}

The authors declare that they have no conflicts of interest.

17. Vaga S, Raimondi MT, Caiani EG et al: Quantitative assessment of intervertebral disc glycosaminoglycan distribution by gadolinium-enhanced MRI in orthopedic patients. Magn Reson Med, 2008; 59: 85-95

18. Lotz JC, Haughton V, Boden SD et al: New treatments and imaging strategies in degenerative disease of the intervertebral disks. Radiology, 2012 264: 6-19

19. Bendtsen M, Bunger CE, Zou X et al: Autologous stem cell therapy main tains vertebral blood flow and contrast diffusion through the endplate in experimental intervertebral disc degeneration. Spine (Phila Pa 1976), 2011; 36: E373-79

20. Wong CS, Yan $\mathrm{CH}$, Gong NJ et al: Imaging biomarker with T1rho and T2 mappings in osteoarthritis - in vivo human articular cartilage study. Eur J Radiol, 2013; 82: 647-50

21. Johannessen W, Auerbach JD, Wheaton AJ et al: Assessment of human disc degeneration and proteoglycan content using T1rho-weighted magnetic resonance imaging. Spine (Phila Pa 1976), 2006; 31: 1253-57

22. Mulligan KR, Ferland CE, Gawri R et al: Axial T1rho MRI as a diagnostic imaging modality to quantify proteoglycan concentration in degenerative disc disease. Eur Spine J, 2014 [Epub ahead of print]

23. Blumenkrantz G, Zuo J, Li $X$ et al: In vivo 3.0-tesla magnetic resonance T1rho and $\mathrm{T} 2$ relaxation mapping in subjects with intervertebral disc degeneration and clinical symptoms. Magn Reson Med, 2010; 63: 1193-200

24. Krause FG, Klammer G, Benneker LM et al: Biochemical T2* MR quantification of ankle arthrosis in pes cavovarus. J Orthop Res, 2010; 28: 1562-68

25. Bittersohl B, Miese FR, Hosalkar HS et al: T2* mapping of hip joint cartilage in various histological grades of degeneration. Osteoarthritis Cartilage, 2012; 20: 653-60

26. Biercevicz AM, Murray MM, Walsh EG et al: $T 2{ }^{*} M R$ relaxometry and ligament volume are associated with the structural properties of the healing ACL. J Orthop Res, 2014; 32: 492-99

27. Shrout PE, Fleiss JL: Intraclass correlations: uses in assessing rater reliability. Psychol Bull, 1979; 86: 420-28

28. Cassinelli EH, Hall RA, Kang JD: Biochemistry of intervertebral disc degeneration and the potential for gene therapy applications. Spine J, 2001; 1 205-14

29. Wang SZ, Rui YF, Lu J, Wang C: Cell and molecular biology of intervertebral disc degeneration: current understanding and implications for potential therapeutic strategies. Cell Prolif, 2014; 47: 381-90

30. Saar G, Zhang B, Ling W et al: Assessment of glycosaminoglycan concentration changes in the intervertebral disc via chemical exchange saturation transfer. NMR Biomed, 2012; 25: 255-61

31. Urban JP, Winlove CP: Pathophysiology of the intervertebral disc and the challenges for MRI. J Magn Reson Imaging, 2007; 25: 419-32

32. Akella SV, Regatte RR, Gougoutas AJ et al: Proteoglycan-induced changes in T1rho-relaxation of articular cartilage at 4T. Magn Reson Med, 2001; 46 419-23 